\title{
Prevalência da síndroma de fragilidade na região norte de Portugal
}

Lisa Teresa Moreira, ${ }^{1}$ Ricardo Torre, ${ }^{2}$ Ana Castro Rollo, ${ }^{1}$ Hugo Silva, ${ }^{3}$ Vasco Duarte, ${ }^{4}$ Maria Antónia Cruz ${ }^{1}$

\section{RESUMO}

Objetivos: Determinar a prevalência da síndroma de fragilidade na população da região Norte de Portugal Continental.

Tipo de estudo: Estudo observacional, descritivo e transversal.

Local: USF Nova Era, USF Veiga do Leça, USF Cristelo, USF São Miguel-O-Anjo.

População: Utentes nascidos antes de 1952 à data da elaboração do estudo.

Métodos: Foi selecionada uma amostra de conveniência de 408 participantes da população de utentes, nascidos antes de 1952, que se encontravam inscritos nas quatro unidades em estudo $(N=7.189)$ em março de 2016 . O número de participantes foi distribuído pelas quatro unidades, de acordo com o seu peso relativo na população [USF Nova Era (132), USF Veiga do Leça (94), USF Cristelo (86) e USF São Miguel-O-Anjo (96)]. Aplicou-se um questionário demográfico, o questionário PRISMA-7 e o Gait Speed Test (para uma distância de quatro metros). Para a análise estatística utilizou-se o programa SPSS ${ }^{\circledR}$, v. 20.

Resultados: A média de idades foi de $74,3 \pm 6,6$, dos quais $54,7 \%$ eram mulheres. A maioria era casada (64,6\%) e com o primeiro ciclo de escolaridade (46,6\%). A prevalência da síndroma de fragilidade foi de $34,5 \%$ e a prevalência de participantes pré-frágeis foi de $18,3 \%$ para a amostra em estudo. A prevalência da síndroma de fragilidade aumenta com a idade, na população não casada e na população que tem menos de quatro anos de escolaridade.

Conclusão: Os resultados obtidos da prevalência da síndroma de fragilidade são superiores aos referidos na literatura europeia e norte-americana, mas semelhantes aos descritos nos países do Sul da Europa.

Palavras-chave: Fragilidade; Geriatria; Idoso frágil.

\section{INTRODUÇÃO}

A abordagem do doente idoso exige cuidados específicos de acordo com a heterogeneidade desta população, às múltiplas comorbilidades, à cronicidade, à dependência, às múltiplas carências (sociais, familiares e económicas), aos estereótipos e à discriminação. A atitude da equipa de saúde deve ser multidisciplinar, integrando a prevenção, o tratamento curativo, a reabilitação, o apoio social e psicológico, com o intuito de promover um envelhecimento saudável e a manutenção da autonomia do doente.

1. USF Nova Era

2. USF Veiga do Leça

3. USF Cristelo

4. USF São Miguel-o-Anjo
É especialmente na medicina geral e familiar que se centram as bases dos cuidados geriátricos. Parte da compreensão destes cuidados relaciona-se com a assimilação de uma das principais síndromas geriátricas, a síndroma de fragilidade (SF).

A SF é uma síndroma médica com múltiplas causas e fatores contribuintes, caracterizada pela diminuição de força, resistência e funções fisiológicas reduzidas que aumentam a vulnerabilidade de o indivíduo desenvolver dependência funcional e/ ou morte. ${ }^{1}$ Na literatura europeia e americana atinge 7 a $10 \%$ da população com mais de 65 anos de idade, podendo atingir 25$-50 \%$ dos indivíduos com mais de 85 anos de idade. . $^{2-5}$ Consiste no aparecimento de alterações fisiológicas (mitocondriais, genéticas e moleculares) que interferem na produção e utilização energética, condicionando uma desregulação multiorgânica. ${ }^{2,6-7}$ 
Esta desregulação multiorgânica é responsável por uma deficiente resposta homeostática a eventos stressores, como alterações ambientais extremas (frio ou calor extremos), doença aguda, cirurgia ou hospitalização. ${ }^{4}$ Nos idosos frágeis, mesmo um evento desestabilizador minor pode desencadear deterioração física e/ou psicológica súbita., ${ }^{2,48}$

O diagnóstico da SF apoia-se no modelo fenotípico introduzido por Fried, em 2001, e compreende a presença de três ou mais de cinco características: fadiga, marcha lentificada, diminuição da atividade física, exaustão e perda de peso não intencional. ${ }^{3,5,8-10} \mathrm{~A}$ soma das características define a categoria em que cada individuo se encontra (Quadro I).

$\mathrm{O}$ doente frágil varia num espectro entre a fase latente de vulnerabilidade, que não é clinicamente aparente na ausência de eventos desestabilizadores, até um estadio terminal com elevado risco de mortalidade precoce. $^{2}$

Os idosos frágeis experienciam maior dificuldade na recuperação da doença, maior risco de quedas, fraturas, hospitalização, deterioração funcional, dependência/institucionalização e maior risco de mortalidade.,4,11

A SF é uma condição crónica e progressiva, cujas fases iniciais mostram responder eficazmente à intervenção precoce, através da implementação de exercícios de resistência que promovem a manutenção da massa e força muscular. A adoção de uma dieta mediterrânea e o aumento da ingestão proteica também mostraram ter benefício. ${ }^{2,4,6-7} \mathrm{~A}$ fase mais grave da SF é irreversível, com um elevado risco de mortalidade nos seis a 12 meses subsequentes, em que o tratamento paliativo parece ser a melhor solução. ${ }^{2}$

A SF distingue-se das múltiplas comorbilidades do doente idoso, na medida em que o mesmo fenótipo surge tanto resultante de processos intrínsecos do envelhecimento, como associado ao estadio final de doenças crónicas que cursam com sarcopenia (cancro, insuficiência cardíaca, doença pulmonar obstrutiva crónica ou infeção pelo vírus da imunodeficiência humana), sugerindo que clinicamente a fragilidade é uma entidade fisiológica per si. ${ }^{2,4,10}$

Tradicionalmente o diagnóstico da SF baseia-se numa avaliação geriátrica; no entanto, a ausência de especialidade em geriatria e a duração prolongada da avaliação geriátrica global questionaram a aplicabilidade

\begin{tabular}{|c|c|}
\hline \multicolumn{2}{|c|}{$\begin{array}{l}\text { QUADRO I. Diagnóstico da SF de acordo com o modelo } \\
\text { fenotípico de Fried }\end{array}$} \\
\hline Classificação & $\begin{array}{l}N^{\circ} \text { de caraterísticas presentes } \\
\text { (fadiga, marcha lentificada, diminuição } \\
\text { da atividade física, exaustão e perda de } \\
\text { peso não intencional) }\end{array}$ \\
\hline Não-frágil & 0 \\
\hline Pré-frágil & $1-2$ \\
\hline Frágil & $3-5$ \\
\hline
\end{tabular}

desta avaliação nos cuidados de saúde primários (CSP). ${ }^{3}$ Nesse sentido, vários instrumentos de avaliação têm sido desenvolvidos para utilização nos CSP, mais fáceis e rápidos de aplicar, como é o caso do SHARE-FI (Frailty Instrument of the Survey of Health, Ageing and Retirement in Europe), TFI (Tilburg Frailty Indicator), GFI (Groningen Frailty Indicator Questionnaire), PRISMA-7 (Program of Research to Integrate Services for the Maintenance of Autonomy), Gait Speed Test e Timed-

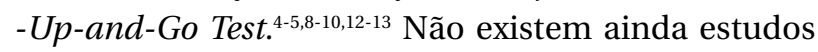
comparativos que permitam aferir qual o teste com maior sensibilidade e especificidade. Atualmente, a British Geriatrics Society recomenda a utilização do Gait Speed Test ou o Timed-Up-and-Go Test, em associação com uma segunda ferramenta como o PRISMA-7 (ferramenta recentemente validada para a população Portuguesa),${ }^{14}$ como forma de aumentar a precisão diagnóstica. ${ }^{4} \mathrm{O}$ Gait Speed Test consiste numa prova de marcha cronometrada $(\mathrm{m} / \mathrm{s})$ para uma distância entre 2,4 e 6 metros, consoante os estudos. Um resultado inferior a $0,8 \mathrm{~m} / \mathrm{s}$ é preditor de $\mathrm{SF}$, associando-se a uma diminuição da sobrevida. ${ }^{3}$ O PRISMA-7 é um questionário que avalia a presença das cinco características fenotípicas da SF através de sete questões. ${ }^{4}$

É objetivo principal dos autores determinar a prevalência da SF na população da região Norte de Portugal Continental e objetivo secundário determinar se a prevalência é influenciada pela idade, género, escolaridade ou estado civil.

\section{MÉTODOS}

Este estudo foi aprovado pela Comissão de Ética para a saúde da Administração Regional de Saúde do Norte (Parecer no 68/2016). 
Efetuou-se um estudo observacional, descritivo e transversal da prevalência da SF, entre 1 de julho e 31 de dezembro de 2016, em quatro Unidades de Saúde Familiar (USF) da zona Norte de Portugal Continental.

Foi selecionada uma população de utentes nascidos antes de 1952, de quatro USF (USF Nova Era, USF Veiga do Leça, USF Cristelo e USF São Miguel-O-Anjo), que se encontravam inscritos em março de 2016, utilizando como critérios de exclusão utentes que não se desloquem ou que o façam em cadeira de rodas. A população em estudo foi de 7.189 utentes (2.316 na USF Nova Era, 1.660 na USF Veiga do Leça, 1.518 na USF Cristelo e 1.695 na USF São Miguel-O-Anjo).

Selecionou-se uma amostra de conveniência, com base no primeiro utente que efetivou inscrição para qualquer tipo de consulta, ao início de cada hora, desde o horário de abertura até ao fecho da unidade e que autorizou, mediante consentimento informado, a participação no estudo. Para garantir a representatividade da amostra selecionaram-se aleatoriamente os dias para a recolha dos dados ao longo dos seis meses subsequentes, até ter-se completado o número de participantes necessários.

O tamanho amostral foi determinado considerando um intervalo de confiança de 95\% e um nível de precisão de $5 \%$, perfazendo um total de 365 participantes. ${ }^{15}$ Tendo em conta os fatores que causam a perda de informação e considerando uma perda amostral de aproximadamente $15 \%$, o tamanho amostral foi aumentado para 408 participantes. O número de participantes foi distribuído pelas quatro unidades em estudo, de acordo com o seu peso relativo na população $(132,94$, 86 e 96 participantes respetivamente).

Foi proposta a participação no estudo a todos os utentes elegíveis pelo secretário clínico. Após este aceitar e assinar consentimento informado, foi encaminhado para uma sala onde se aplicou o questionário demográfico, o questionário PRISMA-7 e onde se realizou a medição do Gait Speed Test, três medições por participante. Os dados recolhidos foram colocados em envelope fechado, garantindo a confidencialidade e anonimato do processo. O envelope foi colocado pelo profissional de saúde em caixa fechada e selada para o efeito.

O questionário demográfico incluiu as variáveis idade (variável quantitativa contínua) e as variáveis sexo, escolaridade e estado civil (variáveis categóricas nominais). O questionário PRISMA-7, recentemente validado para a população portuguesa, ${ }^{14}$ avalia a presença das cinco características fenotípicas da SF através de sete questões (variáveis categóricas nominais).

Gait Speed Test: Prova de marcha cronometrada para uma distância de quatro metros, em que o utente iniciou a marcha um metro antes da marca de partida e terminou um metro após a marca de finalização para que não o tempo inicial de resposta e o tempo de desaceleração final não interferissem na medição. Foram aferidas três medições, tendo-se contabilizado o valor médio para cada participante, em metros por segundo (variável quantitativa contínua).,8 A cronometragem foi elaborada por cada profissional de saúde, utilizando a mesma aplicação eletrónica de cronómetro.

Para a determinação dos indivíduos não frágeis, pré-frágeis e frágeis (SF positivo) utilizaram-se os critérios definidos pela British Geriatrics Society: ${ }^{4}$

- Frágeis, se score no questionário PRISMA-7 $\geq 3$ e Gait Speed Test $<0,8 \mathrm{~m} / \mathrm{s}$.

- Pré-frágeis, se score no questionário PRISMA-7 entre 1 e 2 e Gait Speed Test $<0,8 \mathrm{~m} / \mathrm{s}$.

Para a análise estatística utilizou-se o programa SPSS $^{\circledR}$ v. (64 Bit) 20. Calcularam-se as características demográficas (idade, género, estado civil e escolaridade) da população em estudo, utilizando valor absoluto (percentagem) para as variáveis categóricas e média e desvio-padrão para as variáveis contínuas. Determinou-se a proporção de doentes não-frágeis, pré-frágeis e frágeis (com SF) no total e no grupo etário com mais de 85 anos. Foi avaliada a associação entre a SF e a idade através do $t$-teste e a associação entre a SF e o sexo, o estado civil e a escolaridade utilizando o teste do Qui-quadrado.

\section{RESULTADOS}

Dos 408 participantes todos foram incluídos no estudo. Destes, $54,7 \%$ (223) eram do sexo feminino e $45,3 \%$ (185) do sexo masculino. A média de idades foi de 74,3 $\pm 6,6$ anos e a mediana de 73 anos. De entre os participantes, 9,7\% tinham idade igual ou superior a 85 anos. Relativamente ao estado civil, $4,3 \%$ eram solteiros, $64,6 \%$ casados, $0,3 \%$ viviam em união de facto, $28,9 \%$ eram viúvos, $1,6 \%$ divorciados e $0,3 \%$ separados. Relativamente à escolaridade, $34,2 \%$ dos participantes 
tinham um nível de escolaridade inferior à $4^{\text {a }}$ classe, $46,6 \%$ tinham o $1^{\circ}$ ciclo de escolaridade, $7,8 \%$ o $2^{\circ}$ ciclo de escolaridade, $5,9 \%$ o $3^{\circ}$ ciclo de escolaridade, $3,7 \%$ o ensino secundário e 1,9\% o ensino superior.

No questionário PRISMA-7 obteve-se um total de 231 participantes $(56,6 \%)$ com score entre 1 e 2; e 164 participantes $(40,1 \%)$ com score superior ou igual a 3 (Figura 1). No Gait Speed Test 185 participantes $(45,4 \%)$ alcançaram um valor igual ou superior a $0,8 \mathrm{~m} / \mathrm{seg}$; 223 participantes $(54,6 \%)$ um valor inferior a $0,8 \mathrm{~m} / \mathrm{seg}(\mathrm{Fi}$ gura 2).

$\mathrm{Na}$ amostra em estudo foram identificados, utilizando os critérios descritos, 75 participantes pré-frágeis $(18,3 \%)$ e 141 participantes frágeis/SF positivo $(34,5 \%)$.

Com idade igual ou superior a 85 anos identificaram-se um $(1,3 \%)$ nos indivíduos pré-frágeis e 31 $(22,0 \%)$ nos indivíduos frágeis.

Considerando a amostra de participantes com idade igual ou superior a 85 anos, a presença de indivíduos frágeis é de 79,5\% e na amostra de participantes com idade inferior a 85 anos a presença de indivíduos frágeis é de 29,8\% (Figura 3).

A análise entre as características demográficas e a presença de SF revelou uma associação para a idade, a escolaridade e o estado civil (Quadro II).

\section{DISCUSSÃO}

A prevalência da SF na amostra foi de $34,5 \%$, valor superior ao expectável, considerando os dados do FIT FOR FRAILTY CONSENSUS BEST PRACTICE GUIDANCE FOR THE CARE OF OLDER PEOPLE LIVING WITH FRAILTY IN COMMUNITY AND OUTPATIENT SETTINGS, da British Geriatrics Society ${ }^{4}$ (indivíduos +65 anos: $10 \%$; e +85 anos: $25-50 \%$ ), do FrailTy IN GERIATRICS REVIEW SYLLABUS, da American Geriatrics Society ${ }^{2}$ (indivíduos +65 anos: 7-10\%) e os dados do PREvALENCE OF FRAILTY IN MIDDLE-AGED AND OLDER COMMUNITY-DWELLING EUROPEANS LIVING IN 10 COUNTRIES ${ }^{16}$ (indivíduos +65 anos: 17,0\%). Neste último estudo foi notada uma maior prevalência da SF nos países incluídos do Sul da Europa [Espanha (indivíduos +65 anos: 27,3\%), Itália (indivíduos +65 anos: 23,0\%), França (indivíduos +65 anos: 15,0\%) e Grécia (indivíduos +65 anos: 14,7\%)], assim como uma prevalência maior de indivíduos pré-frágeis $\left(50,9 \%, 45,6 \%, 43,6 \%\right.$ e $44,9 \%$, respetivamente). ${ }^{16}$ No estudo FraILTY PREVALENCE AND SLOW WALKING SPEED IN PERSONS AGE 65 AND OLDER: IMPLICATIONS FOR PRIMARY CARE, ${ }^{3}$

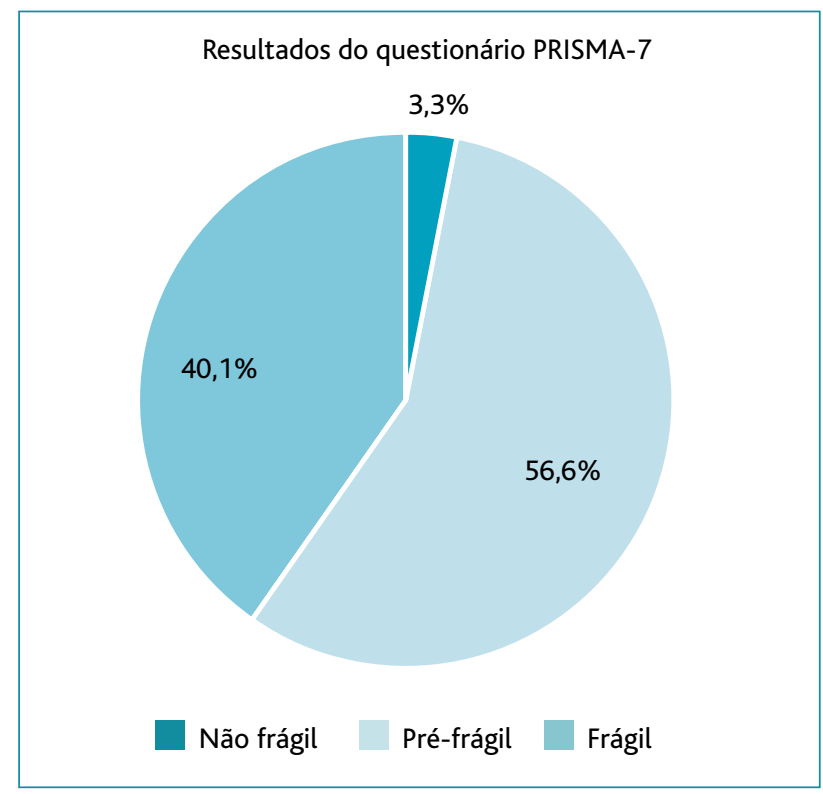

Figura 1. Resultados do questionário PRISMA-7.

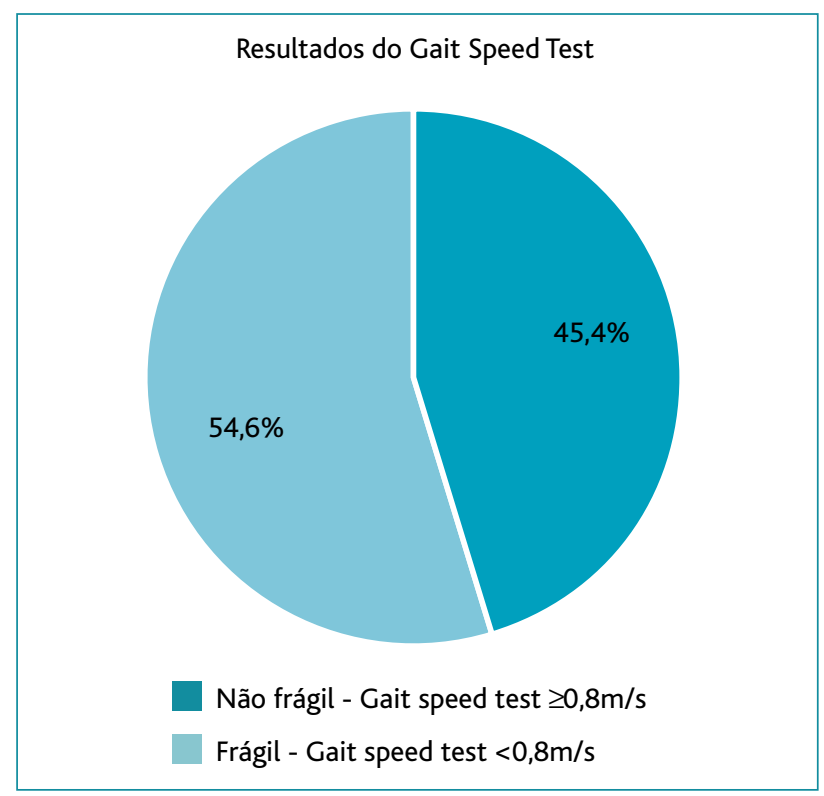

Figura 2. Resultados do Gait Speed Test.

que teve lugar no norte de Madrid, a prevalência da SF foi inferior à encontrada na presente amostra (indivíduos +65 anos: 10,5\%; e +75 anos: 19,1\%). Importa salientar que a diferença nestes dados poderá dever-se à utilização de uma amostra de conveniência de pessoas que recorrem à consulta nas USF em estudo. Tanto no 


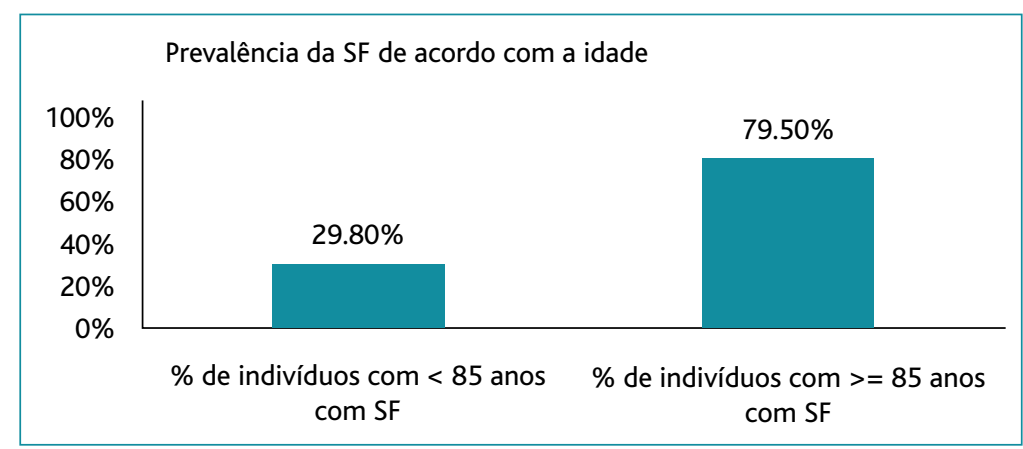

Figura 3. Prevalência da síndroma de fragilidade de acordo com a idade. Legenda: SF = Síndroma de fragilidade.

\section{QUADRO II. Análise comparativa de ambos os grupos de inquiridos}

\begin{tabular}{l|c|c|c} 
Variáveis & Não frágil & Frágil & $\boldsymbol{P}$ \\
\hline Idade & $71,9 \pm 5,2$ & $78,7 \pm 6,6$ & $<0,05$ \\
\hline Sexo & $54,5 \%$ & $55,0 \%$ & 0,938 \\
Feminino & $45,5 \%$ & $45,0 \%$ & \\
Masculino & & & $<0,05$ \\
\hline Escolaridade & $26,5 \%$ & $48,6 \%$ & \\
$<4^{a}$ Classe & $47,4 \%$ & $45,0 \%$ & \\
$1^{\circ}$ Ciclo & $9,5 \%$ & $4,5 \%$ & \\
$2^{\circ}$ Ciclo & $9,0 \%$ & $0,0 \%$ & \\
$3^{\circ}$ Ciclo & $5,2 \%$ & $0,9 \%$ & \\
Secundário & $2,4 \%$ & $0,9 \%$ & \\
Superior & & & $<0,05$ \\
\hline Estado civil & $3,3 \%$ & $6,3 \%$ & \\
Solteiro & $75,4 \%$ & $44,1 \%$ & \\
Casado & $2,4 \%$ & $0,0 \%$ & \\
Divorciado & $0,5 \%$ & $0,0 \%$ & \\
União de facto & $18,0 \%$ & $49,5 \%$ & \\
Viúvo & $0,5 \%$ & $0,0 \%$ & \\
Separado & & & \\
\hline
\end{tabular}

PreVALENCE OF FRAILTY IN MIDDLE-AGED AND OLDER COMMUNITY-DWELLING EUROPEANS LIVING IN 10 COUNTRIES COMO no FRAILTY PREVALENCE AND SLOW WALKING SPEED IN PERSONS AGE 65 AND OLDER: IMPLICATIONS FOR PRIMARY CARE as amostras utilizadas são comunitárias, o que poderá justificar uma prevalência menor.

A relação entre a SF e a idade está bem estabelecida. ${ }^{13,11,16} \mathrm{Na}$ presente amostra também se demonstrou um aumento da prevalência com a idade estatisticamente significativo, com a prevalência a aumentar de $29,8 \%$ nos indivíduos entre os 65 e os 84 anos para $79,5 \%$ naqueles com 85 ou mais anos.

Tal como noutros estudos, na presente amostra também se encontrou uma associação com o sexo feminino, com a população não casada e com a população que tem menos de quatro anos de escolaridade; em todos, esta associação foi estatisticamente significativa, exceto no sexo feminino. ${ }^{1,3,16}$

As limitações do estudo referem-se a aspetos inerentes à seleção da amostra, nomeadamente à exclusão de indivíduos que não se desloquem à USF, como os acamados ou institucionalizados ou aqueles que se deslocam por cadeira de rodas, pela impossibilidade de realizar o Gait Speed Test. De referir ainda a possibilidade de existirem pessoas que cumprem os critérios de seleção e que não frequentam os CSP. Esta possível limitação carece de dados estatísticos.

Relativamente às ferramentas utilizadas, a aplicação do questionário PRISMA-7 teve uma duração média de um minuto por utente e a prova do Gait Speed Test foi realizada numa média de três minutos por utente. Estes dados sugerem a sua aplicabilidade em consulta pelos profissionais de saúde, possibilitando a identificação de pessoas em risco e a promoção de estratégias de intervenção precoce.

Está descrito que o Gait Speed Test e o PRISMA-7 são testes muito sensíveis, mas moderadamente específicos na determinação da $\mathrm{SF}^{3-4}$ razão pela qual a British Geriatrics Society ${ }^{4}$ recomenda a utilização conjunta de ambas as ferramentas como forma de aumentar a especificidade diagnóstica ${ }^{4,8}$ Até à data não foram publicados estudos que utilizem estas duas ferramentas em conjunto, não havendo estudos passíveis de comparação direta.

Os resultados deste estudo permitem refletir sobre os atuais sistemas de apoio à população idosa em Portugal e sobre a necessidade de se implementarem estratégias de intervenção no isolamento social e na promoção do envelhecimento ativo. A identificação dos indivíduos frágeis nas unidades funcionais poderá abrir as portas à implementação de projetos de intervenção, 
recrutando as diversas valências dos CSP e exponenciando a sua prestação individual.

Mais estudos são ainda necessários por forma a determinar a prevalência da SF noutras regiões de Portugal e o seu impacto.

\section{REFERÊNCIAS BIBLIOGRÁFICAS}

1. Morley JE, Vellas B, van Kan GA, Anker SD, Bauer JM, Bernabei R, et al. Frailty consensus: a call to action. J Am Med Dir Assoc. 2013;14(6): 392-7.

2. Fried LP. Frailty. In: Durso SC, Sullivan GM, editors. Geriatrics review syllabus: a core curriculum in geriatric medicine. 8th ed. New York: American Geriatrics Society; 2013. ISBN 9781886775268

3. Castell MV, Sánchez M, Julián R, Queipo R, Martín S, Otero Á. Frailty prevalence and slow walking speed in persons age 65 and older: implications for primary care. BMC Fam Pract. 2013;14:86.

4. Turner G. Fit for frailty: consensus best practice guidance for the care of older people living with frailty in community and outpatient settings. London: British Geriatrics Society; 2014.

5. Romero-Ortuno R, Walsh CD, Lawlor BA, Kenny RA.A frailty instrument for primary care: findings from the Survey of Health, Ageing and Retirement in Europe (SHARE). BMC Geriatr. 2010;10:57.

6. Lucas RW, Kennedy-Malone L. Frailty in the older adult: will you recognize the signs? Nurse Pract. 2014;39(3):28-34.

7. McMillan GJ, Hubbard RE. Frailty in older inpatients: what physicians need to know. QJM. 2012;105(11):1059-65.

8. Turner $G$, Clegg A. Best practice guidelines for the management of frailty: a British Geriatrics Society, Age UK and Royal College of General Practitioners report. Age Ageing. 2014;43(6):744-7.

9. Pialoux T, Goyard J, Lesourd B. Screening tools for frailty in primary health care: a systematic review. Geriatr Gerontol Int. 2012;12(2): 189-97.

10. Romero-Ortuno R, O'Shea D, Kenny RA. The SHARE frailty instrument for primary care predicts incident disability in a European populationbased sample. Qual Prim Care. 2011;19(5):301-9.
11. Gale CR, Cooper C, Sayer AA. Prevalence of frailty and disability: findings from the English Longitudinal Study of Ageing. Age Ageing. 2015;44(1):162-5.

12. Hoogendijk EO, van der Horst HE, Deeg DJ, Frijters DH, Prins BA, Jansen $A P$, et al. The identification of frail older adults in primary care: comparing the accuracy of five simple instruments. Age Ageing. 2013;42(2):262-5.

13. Drubbel I, Bleijenberg N, Kranenburg G, Eijkemans RJ, Schuurmans MJ, de Wit NJ, et al. Identifying frailty: do the Frailty Index and Groningen Frailty Indicator cover different clinical perspectives? A cross-sectional study. BMC Fam Pract. 2013;14:64.

14. Tavares JP, Ferreira AL, Fonseca JP, Barbosa BT, Teixeira AT, Veríssimo MT. Prisma-7 para rastreio da fragilidade em adultos idosos: estudo de validade de conteúdo e fidelidade. Referência. 2016;4(9 Suppl):225.

15. Australian Bureau of Statistics. Sample size calculator [homepage]. Belconnen ACT: ABS; 2016 [updated 2018 Aug 30]. Available from: http://www.abs.gov.au/websitedbs/D3310114.nsf/home/Sample+Size + Calculator

16. Santos-Eggimann B, Cuénoud P, Spagnoli J, Junod J. Prevalence of frailty in middle-aged and older community-dwelling Europeans living in 10 countries. J Gerontol A Biol Sci Med Sci. 2009;64(6):675-81.

\section{CONFLITO DE INTERESSES}

Os autores declaram não ter quaisquer conflitos de interesse.

\section{FINANCIAMENTO}

Os autores declaram que o trabalho não foi objeto de qualquer tipo de financiamento externo.

\section{ENDEREÇO PARA CORRESPONDÊNCIA \\ Lisa Teresa Moreira \\ E-mail: lisamoreira1@gmail.com \\ http://orcid.org/0000-0002-0390-836X}

Recebido em 10-10-2017

Aceite para publicação em 09-09-2018 


\section{ABSTRACT}

\section{PREVALENCE OF FRAILTY SYNDROME IN THE NORTHERN REGION OF MAINLAND PORTUGAL}

Objective: To determine the prevalence of Frailty Syndrome in the population of the northern region of mainland Portugal. Study type: Observational study, descriptive and transversal.

Setting: USF Nova Era, USF Veiga do Leça, USF Cristelo, USF São Miguel-O-Anjo.

Participants: Patients born before 1952 at the time of the study.

Methods: A convenience sample of 408 participants was selected from the population of users born before 1952, enrolled in the family health units included in this study $(N=7,189)$ in March 2016. The number of participants was distributed among the four units, according to their relative weight in the population [USF Nova Era (132), USF Veiga do Leça (94), USF Cristelo (86), and USF São Miguel-O-Anjo (96)]. A demographic questionnaire, the PRISMA-7 questionnaire and the Gait Speed Test (for a distance of four meters) were applied. Statistical analysis was performed using SPSS ${ }^{\circledR}$ v. 20.

Results: The mean age was $74.3 \pm 6.6$, and $54.7 \%$ of the participants were women. Most were married $(64.6 \%)$ and had the first cycle of schooling (46.6\%). The prevalence of frailty syndrome was $34.5 \%$, and the prevalence of pre-frailty was $18.3 \%$ for the study sample. The prevalence of frailty syndrome increases with age, in the unmarried population, and in those with less than four years of schooling.

Conclusion: The prevalence of frailty syndrome is higher than the values reported in European and North American literature, but similar to those described in Southern European countries.

Keywords: Frailty; Geriatrics; Frail elderly. 